\title{
Elektrochemische Abscheidung von Preußisch Blau für die Atemgasanalytik - Methodenvergleich und Schichtcharakterisierung
}

\author{
Thomas J. Rabbow ${ }^{1}$, Rowena Wachtel ${ }^{1}$, Alexander Michaelis ${ }^{1}$, Joanna Konat-Stepowicz ${ }^{2}$, Rüdiger \\ Eichler $^{2}$, Manfred Decker ${ }^{3}$, Winfried Vonau ${ }^{3}$ \\ ${ }^{1}$ Fraunhofer IKTS, Dresden, Winterbergstraße 28, 01277 Dresden, \\ ${ }^{2}$ FILT GmbH, Robert-Rössle-Straße 10, Haus 55, 13125 Berlin \\ ${ }^{3}$ Kurt-Schwabe-Institut, Kurt-Schwabe-Straße 4, 04720 Ziegra-Knobelsdorf
}

\section{Zusammenfassung}

Die elektrochemische Abscheidung von Goldnanopartikeln und Schichten aus Preußisch Blau ermöglicht den nicht-enzymatischen Nachweis von Wasserstoffperoxid im nanomolaren Konzentrationsbereich. Zur Erzielung besonders hoher Empfindlichkeiten hat sich die potentiostatische Abscheidung dünner Preußisch Blau Schichten als günstig erwiesen. Als Kriterium für eine hohe Sensitivität des Mediators gegenüber Wasserstoffperoxid kann ein möglichst geringer Peakabstand im Zyklovoltammogramm herangezogen werden, der ein reversibles Verhalten für die Oxidation und Reduktion des abgeschiedenen Preußisch Blau ausdrückt. Die Bedingungen für eine hohe Reproduzierbarkeit der Schichten werden evaluiert.

\section{Preußisch Blau Abscheidung}

Wasserstoffperoxid wird als Marker für die Früherkennung entzündlicher Prozesse in der Lunge ein großes Potential zugesprochen. Für die Atemgasanalytik muss ein Sensor entwickelt werden, der auch bei niedrigen Temperaturen, die eine Kondensation des Exhalats zulassen, sicher und mit hoher Empfindlichkeit arbeitet. Kommerziell verfügbare Sensoren auf enzymatischer Basis sind aufgrund der verminderten enzymatischen Aktivität unterhalb des physiologischen Temperaturbereichs nicht geeignet.

Für einen nicht-enzymatischen Nachweis von Wasserstoffperoxid ist die Verbindung Preußisch Blau $\left(\mathrm{Fe}^{\mathrm{III}}{ }_{4}\left[\mathrm{Fe}^{\mathrm{II}}(\mathrm{CN})_{6}\right]_{3}\right)$ als Mediator seit den achtziger Jahren bekannt [1]. Es werden verschiedene elektrochemische Verfahren für die Abscheidung (z.B. Zyklovoltammetrie, potentiostatische und galvanostatische Abscheidungen) beschrieben. Der analytische Nachweis beruht auf der chemischen Oxidation der Eisen(II)-Ionen im Preußisch Blau (PB) Kristall durch das Wasserstoffperoxid, welches hierdurch indirekt über die anschließende elektrochemische Reduktion der gebildeten Eisen(III)-Ionen in der Mediatorschicht quantitativ erfassbar wird [2].

$2 \mathrm{H}^{+}+\mathrm{K}_{2} \mathrm{Fe}_{4}\left[\mathrm{Fe}(\mathrm{CN})_{6}\right]_{3} \cdot \mathrm{xH}_{2} \mathrm{O}+\mathrm{H}_{2} \mathrm{O}_{2} \rightarrow \mathrm{Fe}_{4}\left[\mathrm{Fe}(\mathrm{CN})_{6}\right]_{3} \cdot \mathrm{xH}_{2} \mathrm{O}+2 \mathrm{~K}^{+}+2 \mathrm{H}_{2} \mathrm{O}$

Für eine hohe Empfindlichkeit muss sowohl ein hoher Umsatz zwischen dem Preußisch Blau und dem Wasserstoffperoxid gewährleistet sein, als auch eine genügende Leitfähigkeit innerhalb des anorganischen Halbleiters bestehen, um einen Ladungsträgertransport vom Mediator auf das Sensorsubstrat sicher zu stellen. Hierzu gilt es beispielsweise, ein Optimum für die Dicke der PB-Schicht zu finden, da besonders starke Schichten zwar in hohem Maße mit dem Wasserstoffperoxid reagieren, jedoch keine ausreichende Leitfähigkeit besitzen und folglich eine schlechte Empfindlichkeit aufweisen.

Die elektrochemische PB-Bildung stellt eine Kopplung eines elektrochemischen und eines rein chemischen Schrittes (eine Fällungsreaktion) dar. Elektrochemisch wird das Hexacyanoferrat reduziert und bildet mit dem freien Eisen lösliches PB, welches schließlich mit weiterem freien Hexacyanoferrat zum PB reagiert. $4 \mathrm{Fe}^{3+}+3 \mathrm{Fe}(\mathrm{CN})_{6}^{4-} \rightarrow \mathrm{Fe}_{4}\left[\mathrm{Fe}(\mathrm{CN})_{6}\right]_{3}$

Gl. 2

Neben der Sicherstellung eines robusten Abscheidungsverfahrens, durch welches stets gleiche Mengen von PB erzeugt werden, sollte auch eine optimale Mikrostruktur bzw. Morphologie des PB erzeugt werden. Beim Nachweis muss das Wasserstoffperoxid an der möglichst großen Oberfläche des PB reagieren und das zweiwertige Eisen zum dreiwertigen oxidieren. Des Weiteren muss für einen quantitativen Nachweis jedes $\mathrm{H}_{2} \mathrm{O}_{2}$-Molekül mit den Eisenatomen umgesetzt werden, was durch eine hohe PB-Grenzfläche zum Elektrolyten erreicht werden kann. Hiermit sind die Bedingungen für die Bildung von PB zum hochempfindlichen $\mathrm{H}_{2} \mathrm{O}_{2}$-Nachweis formuliert. 
Das Sensorprinzip stellt ein klassisches Beispiel für eine Elektrokatalyse dar, bei dem das PB den Elektronenfluss zwischen $\mathrm{H}_{2} \mathrm{O}_{2}$ und Elektroden stark beschleunigt. Für carbonhaltige Elektrodensubstrate ist jedoch auch der Elektronenfluss zwischen PB und Substrat gehemmt. Durch die Abscheidung von AuNanopartikeln verbessert sich der Elektronenübergang zwischen dem PB und dem graphithaltigen Substrat [3]. In der Abbildung 1 sind siebgedruckte Carbonelektroden (Graphit-Ruß-Mischungen, Gwent) gezeigt, die elektrochemisch mit Goldnanopartikeln beschichtet wurden. Zu dicke Schichten eines PB-Kristalls führen zum Aufreißen der Schicht (Abb. 1 rechts). Die modifizierten Elektroden hatten eine Fläche von 0,25 cm². Als Referenzelektrode diente eine Kalomelelektrode. Sofern nicht anders angegeben, wurde das PB aus einer Mischung von 2,5 $\mathrm{mM} \mathrm{K}_{3}\left[\mathrm{Fe}(\mathrm{CN})_{6}\right]$ und 2,5 $\mathrm{mM} \mathrm{FeCl}_{3}$ in $0,1 \mathrm{M} \mathrm{KCl}$ hergestellt.

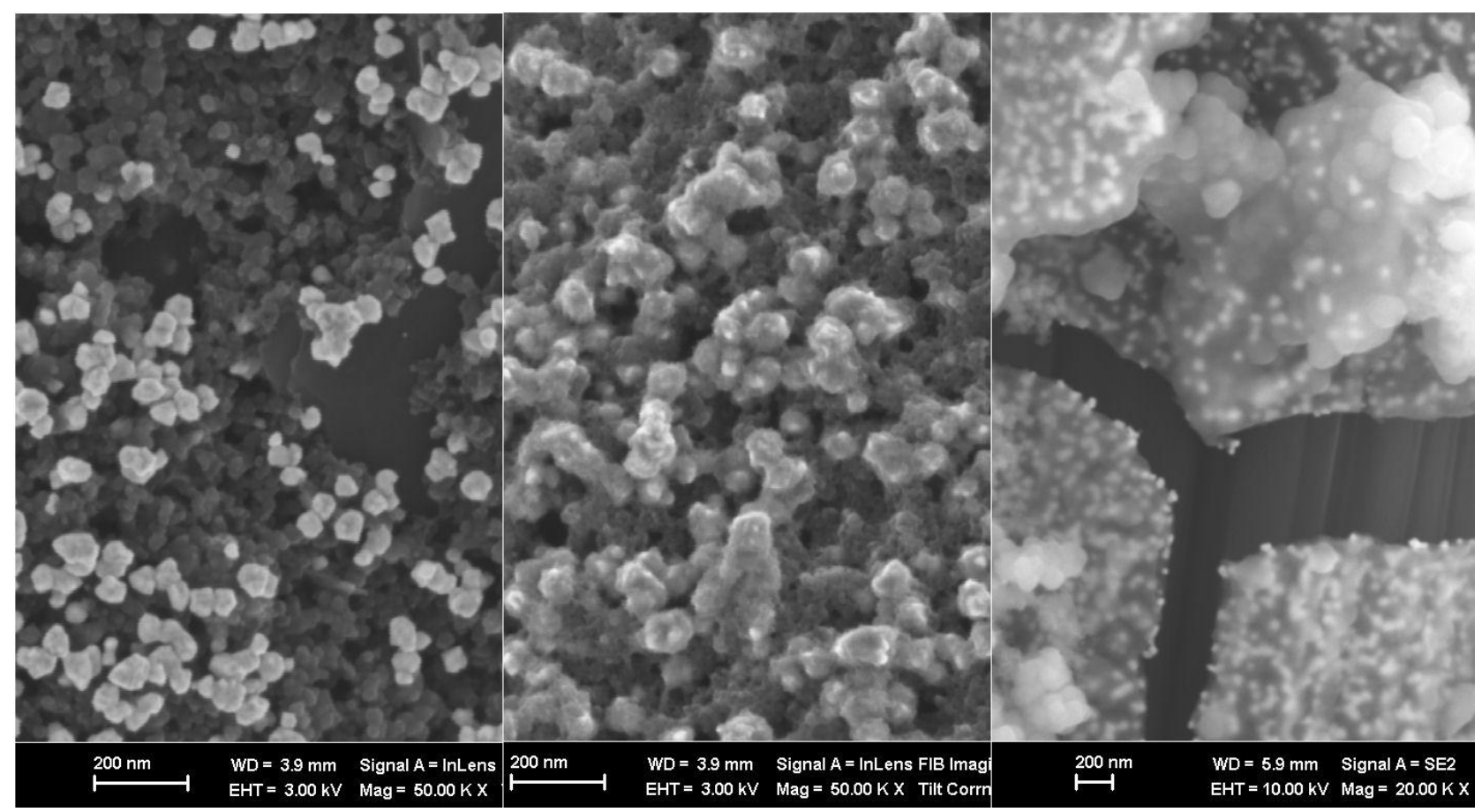

Bild 1 Links: Au-Nanopartikel auf Carbon-Siebdruckelektrode. Mitte: sehr dünne PB-Schicht auf Au-Nanopartikeln. Rechts: Zu starke PB-Schicht, die ein Aufreißen der Schicht bewirkt

\subsection{Ladungsmenge - Schichtaktivität}

Preußisch Blau ermöglicht den $\mathrm{H}_{2} \mathrm{O}_{2}$-Nachweis indem es als Elektrokatalysator wirkt. Für eine möglichst hohe Reproduzierbarkeit der Schichten wird die potentiostatische Abscheidung mit gleichzeitiger Kontrolle der Ladungsmenge gewählt. Hierdurch lässt sich der elektrochemische Reduktionsprozess auswählen und die Menge des Umsatzes festlegen. In Abbildung 2 (links) ist die Reduktion des Hexacyanoferrats bei $\mathrm{E}=50 \mathrm{mV}_{\mathrm{SCE}}$ gezeigt. Der Verlauf des Reduktionsstroms ist für vier verschiedene Ladungsmengen nahezu identisch, lediglich die Abscheidungsdauer erhöht sich mit der Ladungsmenge. Die Zyklovoltammogramme in Abb. 2 (rechts) zeigen, dass die gebildet Menge an PB mit der Ladungsmenge zunimmt sowie auch der Peakabstand (vgl. Tafel 1). Bei $\mathrm{Q}=-21 \mathrm{mC}$ wird jedoch ein geringerer Strom als bei $\mathrm{Q}=-14 \mathrm{mC}$ beobachtet, d. h. ein gewisser Teil des reduzierten Blutlaugensalzes wird nicht in Preußisch Blau an der Elektrodenoberfläche umgewandelt. Sowohl das freie Eisen als auch die Hexacyanoferrate unterliegen einem ständigen Diffusionsprozess. Dies bewirkt, dass zwar die Ladungsmenge der elektrochemischen Reduktion überwacht werden kann, hieraus jedoch nicht eindeutig auf die an der Elektrode angelagerte Masse des PB geschlossen werden kann. Ein Teil des umgesetzten Hexacyanoferrates kann durch Diffusion zu einer Fällung von PB im Elektrolyten führen. Dieser Teil des Umsatzes steht für die Beschichtung und die Sensoranwendung nicht mehr zur Verfügung. Der beschriebene Vorgang ist dafür verantwortlich, dass auch unter Einhaltung konstanter Abscheidungsbedingungen Schwankungen in der Empfindlichkeit der Sensoren beobachtet werden. Eine ideale, hochempfindliche Sensorschicht zeigt einen sehr geringen Peakabstand und gleichzeitig einen hohen Peakstrom, d. h. eine hohe Oberfläche und eine reversible Redoxreaktion. Im Folgenden soll Erhöhung der PB-Menge auf den Sensoren unter Beibehaltung eines geringen Peakabstandes untersucht werden. 

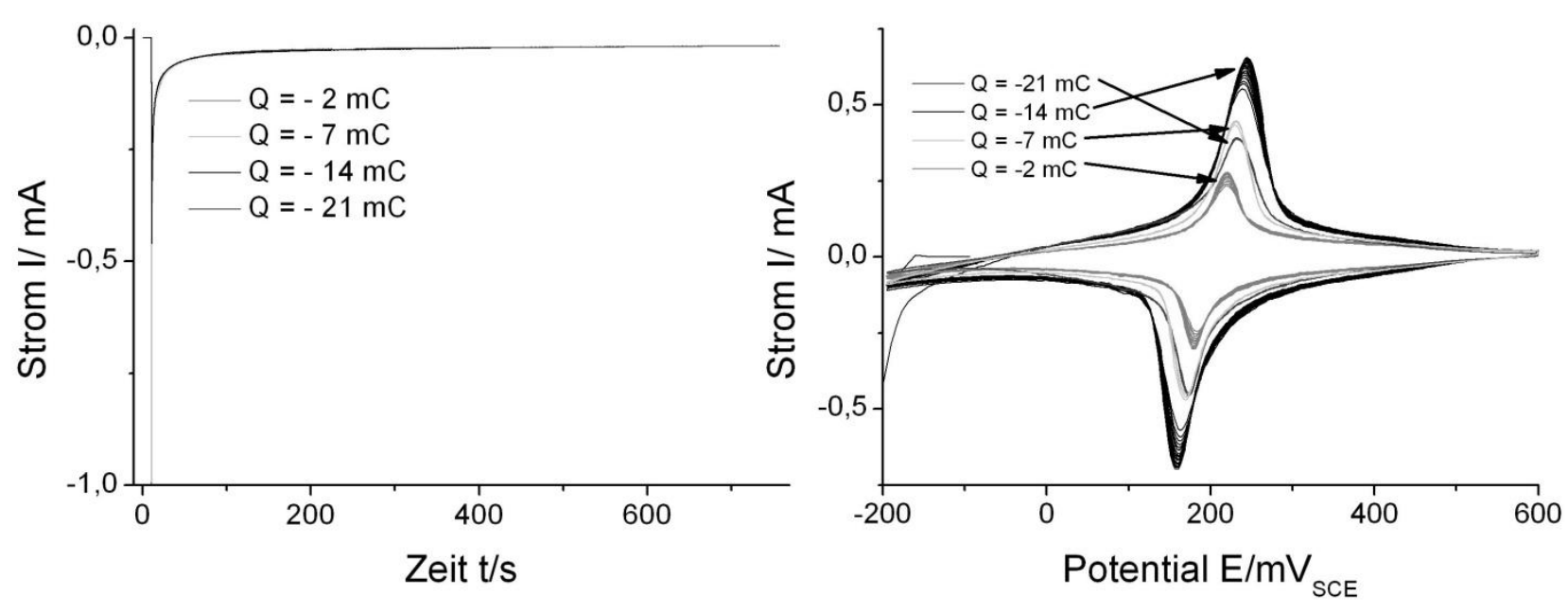

Bild 2 Links: Stromverlauf während der Hexacyanoferrat-Reduktion und PB-Abscheidung mit verschiedenen Ladungsmengen. Rechts: Zyklovoltammogramme der gebildeten PB-Schichten (in 0,1 M KCl, pH = 3)

Tafel 1 Peakströme und Peakabstände in Abhängigkeit von der geflossenen Ladungsmenge

\begin{tabular}{c|c|c}
\hline Ladungsmenge Q/mC & Anodischer Peakstrom $\mathrm{I}_{\mathrm{p}} / \mathrm{mA}$ & Peakabstand $\Delta \mathrm{E}_{\mathrm{p}} / \mathrm{mV}$ \\
\hline-2 & 0,28 & 40 \\
\hline-7 & 0,45 & 62 \\
\hline-14 & 0,65 & 85 \\
\hline-21 & 0,39 & 58 \\
\hline
\end{tabular}

\subsection{Einfluss der Eduktkonzentration auf die Schichtbildung und den $\mathrm{H}_{2} \mathrm{O}_{2}$-Nachweis}

Zur Erzielung einer höheren Preußisch Blau Bildung an der Elektrode und einer Verringerung der Fällung im Elektrolyten kann die Eisen(III)-chlorid Konzentration erhöht werden, sodass das Löslichkeitsprodukt des PB während der Reduktion des Hexacyanoferrats schneller erreicht wird (vgl. Gl. 2). In Abbildung 3 (links) sind vier Zyklovoltammogramme von PB-Schichten gezeigt, die bei gleicher geflossener Ladungsmenge $(\mathrm{Q}=-14 \mathrm{mC})$ hergestellt wurden, d.h. es wurde immer dieselbe Menge Hexacyanoferrat reduziert. Variiert wurde das Konzentrationsverhältnis vom Hexacyanoferrat(III) zum Eisen(III)-chlorid von 1:1 bis 1:4 bzw. von jeweils 2,5 mM auf 2,5 mM / $10 \mathrm{mM}$. Deutliche Unterschiede sind im Peakstrom und dem Peakabstand der abgeschiedenen Schichten festzustellen (vgl. Tafel 2). Durch die Erhöhung der Eisen(III)-chloridKonzentration wird erheblich mehr PB an der Elektrode gebildet. Die Schichten zeigen jedoch eine verringerte Leitfähigkeit, was sich in der Erhöhung des Peakabstandes $\Delta \mathrm{E}_{\mathrm{p}}$ äußert. Ein hoher Peakabstand bedeutet eine verminderte elektrochemische Reaktivität bzw. einen erhöhten Ladungsdurchtrittswiderstand, d. h. diese Schichten sind für den $\mathrm{H}_{2} \mathrm{O}_{2}$-Nachweis weniger geeignet. Bei einem Konzentrationsverhältnis von 1:4 wird dagegen die geringste Menge an PB festgestellt. Es kann derzeit nur vermutet werden, dass die Schicht in diesem Fall eine zu geringe Haftung an das Substrat aufweist und sich nach der Bildung wieder ablöst.

Zum Test der Empfindlichkeit der Schichten wurde dreimal $50 \mu \mathrm{L}$ mit $245 \mathrm{nmol} \mathrm{H}_{2} \mathrm{O}_{2}$ zu der Lösung gegeben $(4 \mathrm{~mL} 0,1 \mathrm{M} \mathrm{KCl}, \mathrm{pH}=3)$. Bei einer Polarisation der Elektroden auf $\mathrm{E}=50 \mathrm{mV}_{\mathrm{SCE}}$ ergibt sich mit der Zugabe von $\mathrm{H}_{2} \mathrm{O}_{2}$ ein kathodischer Strompeak. In Abbildung 3 (rechts) ist ersichtlich, dass die höchste Empfindlichkeit an Schichten beobachtet wird, die aus eine 1:1 Mischung der Eisensalze hergestellt wurden. Die Schichten aus der 1:2 und 1:3 Mischung zeigen einen geringen Strom beim Wasserstoffperoxidnachweis bzw. erhebliche Schwankungen in der Peakhöhe. Mit der Änderung des Verhältnisses vom Hexacyanoferrat(III) zum Eisen(III)-chlorid von 1:1 auf 1:4 erhöht sich zudem das Rauschen des Grundstromes. 

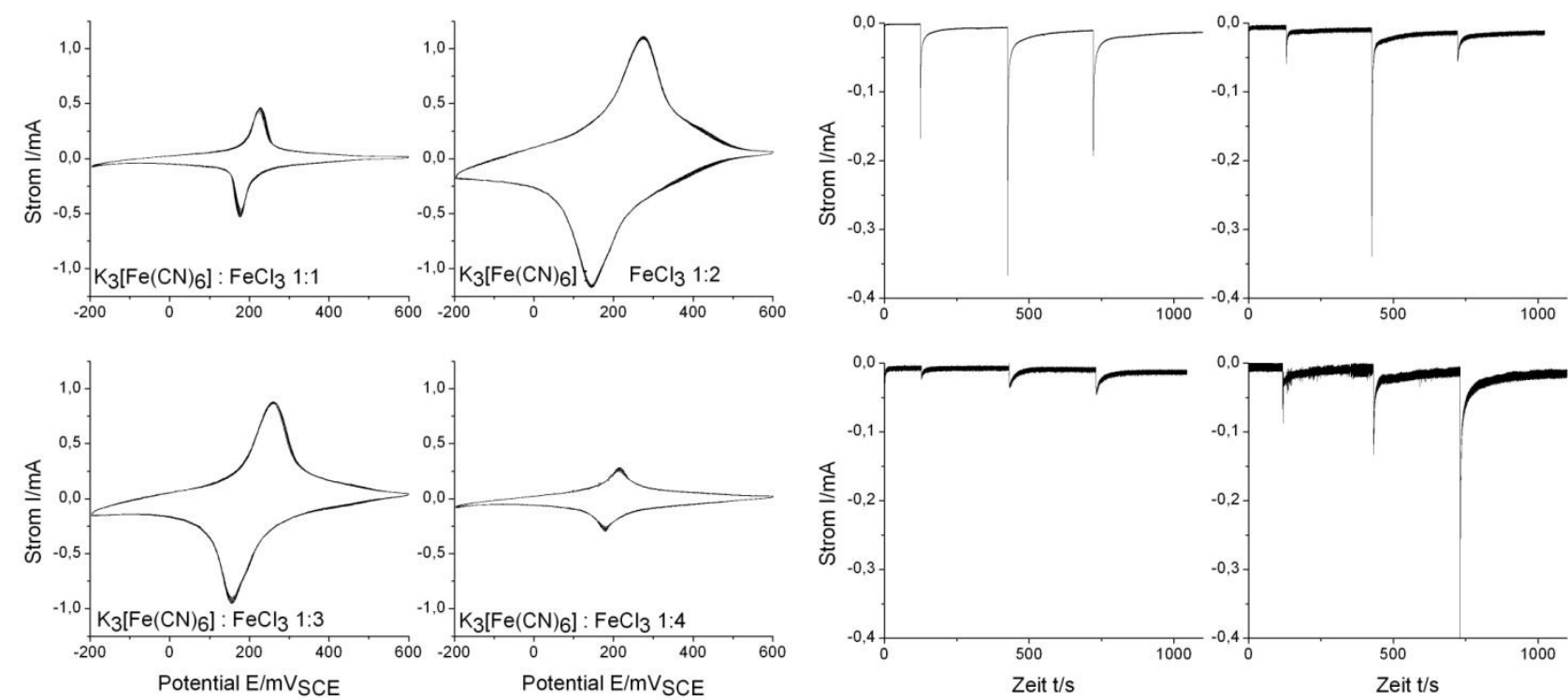

Bild 3 Links: Zyklovoltammogramme von PB-Schichten gebildet aus Lösungen mit unterschiedlicher Konzentration von $\mathrm{K}_{3} \mathrm{Fe}(\mathrm{CN})_{6}$ und $\mathrm{FeCl}_{3}$. Rechts: $\mathrm{H}_{2} \mathrm{O}_{2}$-Nachweis (je 3x245 nmol in $4 \mathrm{~mL} \mathrm{0,1} \mathrm{M} \mathrm{KCl,} \mathrm{pH=3)} \mathrm{an} \mathrm{den} \mathrm{PB-Schichten}$

Tafel 2 Abscheidungsdauer, Peakstrom und Peakabstand bei versch. Konzentrationsverhältnissen $\mathrm{K}_{3}\left[\mathrm{Fe}\left(\mathrm{CN}_{6}\right]: \mathrm{FeCl}_{3}\right.$ während der potentiostatischen Abscheidung

\begin{tabular}{c|c|c|c|}
\hline Konzentrationsverhältnis & Abscheidungsdauer t/s & Peakstrom Ip/mA & Peakabstand $\Delta \mathrm{E}_{\mathrm{p}} / \mathrm{mV}$ \\
\hline $1: 1$ & 431 & 0,39 & 60 \\
& - & 0,46 & 52 \\
\hline $1: 2$ & 538 & 0,80 & 113 \\
& 544 & 1,12 & 83 \\
\hline $1: 3$ & 759 & 0,58 & 113 \\
\hline $1: 4$ & 653 & 0,88 & 38 \\
\hline
\end{tabular}

Mit Hilfe von Messungen mit einer Quarz-Mikrowaage soll die elektrochemische PB-Bildung in Abhängigkeit von der Lösungszusammensetzung und den Abscheidungsbedingungen nachvollzogen werden, sodass der Reduktionsstrom eindeutig mit der BP-Bildung korreliert werden kann. Hieraus sollen Bedingungen ermittelt werden, die es ermöglichen, gerade so viel PB aufzubauen, dass die Grenzfläche der Schichten maximiert und der Ladungsdurchtrittswiderstand minimiert wird.

\section{Literatur}

[1] F. Ricci, G. Palleschi, Biosens. Bioelectron., 2005, 389-407

[2] I.L. De Mattos, 1: Gorton; T. Ruzgas, Biosens. Bioelectron., 2003, 193-200

[3] S.S. Kumar, J. Joseph, K. L. Phani, Mater. Chem., 2007, 4722-4730

Die Entwicklung in Zusammenarbeit mit dem Kurt-Schwabe-Institut für Mess- und Sensortechnik e.V. Meinsberg und der FILT GmbH, Berlin-Buch, wird von der AiF im Rahmen des Programms ,Zentrales Innovationsprogramm Mittelstand“" (ZIM) unter dem Kennzeichen KF 2087309AJ9 gefördert. Die Autoren danken für die Unterstützung. 